\title{
Use of antipsychotic drugs and cholinesterase inhibitors and risk of falls and fractures: self-controlled case series
}

\author{
Grace Hsin-Min Wang, ${ }^{1}$ Kenneth K C Man, ${ }^{2}$ Wei-Hung Chang, ${ }^{3,4}$ Tzu-Chi Liao, ${ }^{1}$ \\ Edward Chia-Cheng Lai ${ }^{1}$
}

${ }^{1}$ School of Pharmacy, Institute of Clinical Pharmacy and

Pharmaceutical Sciences, College of Medicine, National Cheng

Kung University, Tainan, Taiwan

${ }^{2}$ Research Department of

Practice and Policy, UCL School

of Pharmacy, London, UK

${ }^{3}$ Department of Psychiatry,

National Cheng Kung University

Hospital, College of Medicine,

National Cheng Kung University,

Tainan, Taiwan

${ }^{4}$ Department of Psychiatry,

National Cheng Kung University

Hospital, Dou-Liou Branch,

Yunlin, Taiwan

Correspondence to: EC-C Lai edward lai@mail.ncku.edu.tw (ORCID 0000-0002-5852-7652) Additional material is published online only. To view please visit the journal online.

Cite this as: BMJ 2021;374:n1925 http://dx.doi.org/10.1136/bmj.n1925

Accepted: 27 July 2021

\section{ABSTRACT}

\section{OBJECTIVE}

To evaluate the association between the use of antipsychotic drugs and cholinesterase inhibitors and the risk of falls and fractures in elderly patients with major neurocognitive disorders.

DESIGN

Self-controlled case series.

SETTING

Taiwan's National Health Insurance Database.

\section{PARTICIPANTS}

15278 adults, aged $\geq 65$, with newly prescribed antipsychotic drugs and cholinesterase inhibitors, who had an incident fall or fracture between 2006 and 2017. Prescription records of cholinesterase inhibitors confirmed the diagnosis of major neurocognitive disorders; all use of cholinesterase inhibitors was reviewed by experts.

\section{MAIN OUTCOME MEASURES}

Conditional Poisson regression was used to derive incidence rate ratios and $95 \%$ confidence intervals for evaluating the risk of falls and fractures for different treatment periods: use of cholinesterase inhibitors alone, antipsychotic drugs alone, and a combination of cholinesterase inhibitors and antipsychotic drugs, compared with the non-treatment period in the same individual. A 14 day pretreatment period was defined before starting the study drugs because of concerns about confounding by indication.

RESULTS

The incidence of falls and fractures per 100 person years was 8.30 (95\% confidence interval 8.14 to 8.46) for the non-treatment period, 52.35 (48.46 to 56.47) for the pretreatment period, and 10.55 (9.98 to 11.14 ), 10.34 (9.80 to 10.89 ), and 9.41 (8.98

\section{WHAT IS ALREADY KNOWN ON THIS TOPIC}

Antipsychotic drugs and cholinesterase inhibitors have been reported to increase the incidence of falls and fractures in patients with major neurocognitive disorders

Confounding by indication should be considered when evaluating the association between drugs and adverse reactions because cognitive impairment and neuropsychiatric symptoms of major neurocognitive disorders might lead to a high risk of falls and fractures

\section{WHAT THIS STUDY ADDS}

The risk of falls and fractures was highest before patients started treatment with cholinesterase inhibitors and antipsychotic drugs, implying that factors other than the use of drugs might have affected the incidence of falls and fractures Although the high risk of falls and fractures in the pretreatment period was reduced after patients received treatment, the results indicated that patients might not have regained a stable condition of their major neurocognitive disorder to 9.86) for use of a combination of cholinesterase inhibitors and antipsychotic drugs, antipsychotic drugs alone, and cholinesterase inhibitors alone, respectively. Compared with the non-treatment period, the highest risk of falls and fractures was during the pretreatment period (adjusted incidence rate ratio $6.17,95 \%$ confidence interval 5.69 to 6.69 ), followed by treatment with the combination of cholinesterase inhibitors and antipsychotic drugs $(1.35,1.26$ to 1.45$)$, antipsychotic drugs alone $(1.33,1.24$ to 1.43$)$, and cholinesterase inhibitors alone $(1.17,1.10$ to 1.24$)$.

\section{CONCLUSIONS}

The incidence of falls and fractures was high in the pretreatment period, suggesting that factors other than the study drugs, such as underlying diseases, should be taken into consideration when evaluating the association between the risk of falls and fractures and use of cholinesterase inhibitors and antipsychotic drugs. The treatment periods were also associated with a higher risk of falls and fractures compared with the non-treatment period, although the magnitude was much lower than during the pretreatment period. Strategies for prevention and close monitoring of the risk of falls are still necessary until patients regain a more stable physical and mental state.

\section{Introduction}

Older adults with major neurocognitive disorders are often considered vulnerable and prone to falls and related fractures. ${ }^{1}$ Falls and fractures are the most common causes for admission to hospital in older adults and are associated with substantial morbidity and mortality. ${ }^{2}$ Cholinesterase inhibitors are typically used to improve cognition and might be linked to the risk of falls and fractures as a result of the syncope caused by parasympathomimetic effects. ${ }^{3-5}$ Antipsychotic drugs are commonly prescribed to treat neuropsychiatric symptoms in patients with major neurocognitive disorders, ${ }^{6}$ and concerns have been raised about falls and fractures. ${ }^{7}$ Because of the potential adverse effects of antipsychotic drugs, including orthostatic hypotension, sedation, blurred vision, and extrapyramidal symptoms, the United States Food and Drug Administration has suggested that a complete assessment of the risk of falls should be undertaken before the start of treatment. ${ }^{8}$ Beers criteria ${ }^{9}$ and other studies ${ }^{10-22}$ have also suggested that antipsychotic drugs might be associated with the risk of falls and fractures.

Although several studies and guidelines have suggested that cholinesterase inhibitors and antipsychotic drugs might be associated with the risk of falls and fractures, other studies reached different conclusions. Jin et al and Kim et al found no association 
between the use of cholinesterase inhibitors and the incidence of falls and fractures in patients with major neurocognitive disorders. ${ }^{23}{ }^{24}$ One systematic review and meta-analysis and an observational study also found that antipsychotic drugs were not associated with the risk of falls and fractures. ${ }^{24} 25$ Confounding by indication could partly explain the conflicting results from these studies. For example, patients with neuropsychiatric symptoms of major neurocognitive disorders might manifest depression, irritability, agitation, and hallucinations that could lead to the prescription of antipsychotic drugs, and both the symptoms and the treatments could increase the risk of subsequent falls and fractures. This confounding effect is especially likely when the events are seen within a short period of time before patients with major neurocognitive disorders begin treatment of their neuropsychiatric symptoms. But only a few studies evaluating the association between cholinesterase inhibitors, antipsychotic drugs, and the risk of falls and fractures have looked at this issue. ${ }^{1826}$

With an ageing society, the incidence of major neurocognitive disorders and associated neuropsychiatric symptoms is increasing. ${ }^{27}$ Although the use of antipsychotic drugs might be associated with falls and fractures, as reported by the FDA and Beers criteria, antipsychotic drugs can still be prescribed to control neuropsychiatric symptoms in patients with major neurocognitive disorders. Therefore, it has become increasingly important to understand the risk profiles of patients receiving cholinesterase inhibitors and antipsychotic drugs to prevent falls and fractures from occurring. We evaluated the associated risk of falls and fractures in patients receiving both cholinesterase inhibitors and antipsychotic drugs. Specifically, we assessed the risk of falls and fractures during the period before treatment to understand whether the risk arose predominantly from the use of the drugs or from the underlying diseases that required treatment.

\section{Methods}

\section{Data sources}

We used data from the National Health Insurance Database, 2003-2017, provided by the Health and Welfare Data Science Centre, Taiwan. Details of the database have been described elsewhere. ${ }^{28}$ Briefly, the National Health Insurance Database is derived from the National Health Insurance programme of Taiwan, and contains the records of about 23 million people (nearly $99.9 \%$ of the total population of Taiwan). The National Health Insurance Database includes records of diagnoses, drugs, and procedures from outpatient, inpatient, and emergency departments, and from contracted pharmacies. Many major disease diagnoses in the database have been validated by previous studies, including ischaemic stroke, ${ }^{29}$ epilepsy, ${ }^{30}$ hypertension, ${ }^{31}$ diabetes, ${ }^{31}$ hyperlipidaemia, ${ }^{31}$ coronary artery disease, ${ }^{31}$ atrial fibrillation, ${ }^{31}$ heart failure, ${ }^{32}$ Parkinson's disease, ${ }^{33}$ major neurocognitive disorders, ${ }^{33}$ schizophrenia, ${ }^{34}$ bipolar disorder, ${ }^{34}$ and depression. ${ }^{34}$ The diagnosis codes for osteoarthritis, ${ }^{35}$ osteoporosis, ${ }^{35}$ cataract, ${ }^{35}$ falls, ${ }^{36}$ and fractures ${ }^{35}$ have not been validated but were selected based on previous studies and from the expert opinions of a psychiatrist and a geriatrician. Cholinesterase inhibitors and antipsychotic drugs are mostly reimbursed by the National Health Insurance programme in Taiwan, meaning that most prescription records have been captured. Also, we linked the National Health Insurance Database to the Cause of Death registry data to precisely identify patients who died during the study period.

\section{Study population}

The study period was 2006-2017. We selected adults aged $\geq 65$ on 1 January 2006 who had received at least one prescription of both antipsychotic drugs and cholinesterase inhibitors for neuropsychiatric symptoms of major neurocognitive disorders, and who had at least one fall or fracture during the study period. The prescription records for cholinesterase inhibitors were used to confirm the diagnoses of major neurocognitive disorders because experts from the National Health Insurance Administration reviewed all use of cholinesterase inhibitors, based on the Diagnostic and Statistical Manual of Mental Disorders, fifth edition, and patient scores on the mini-mental state examination. We did not consider low dose sulpiride (50 mg/day) as an antipsychotic drug because of its frequent use for gastroduodenal ulcers; prochlorperazine is more widely used as an antiemetic in Taiwan and was also excluded as an antipsychotic drug. We excluded patients with a record of an antipsychotic drug or cholinesterase inhibitor, or with a fall or fracture in 2003-2005 (washout period) to ensure that only new users of the study drugs with no history of falls or fractures were included in the study population. We also excluded patients with underlying schizophrenia or bipolar disorder to ensure that antipsychotic drugs were used to treat the neuropsychiatric symptoms of major neurocognitive disorders. Figure 1 shows a flowchart of the selection of the study population.

\section{Study design}

We applied the self-controlled case series design in this study. ${ }^{37}$ Self-controlled case series include participants who have both the outcome and the treatment of interest during a prespecified study period. These participants act as their own control, and thus all time constant covariates varying between individuals are controlled. Self-controlled case series enable risk estimates by comparing the incidence rates of the outcome between the non-treatment and treatment periods, based on the conditional Poisson regression model.

\section{Outcome events and treatment periods}

The primary outcome was a combination of falls (ICD-9 (international classification of diseases, ninth edition) codes E880-E888; ICD-10 (international classification of diseases, 10th edition) codes W00-W19) and traumatic fractures (ICD-9 codes 800-829; ICD-10 codes S02, S12, S22, S32, S42, S52, S62, S72, S82, and 


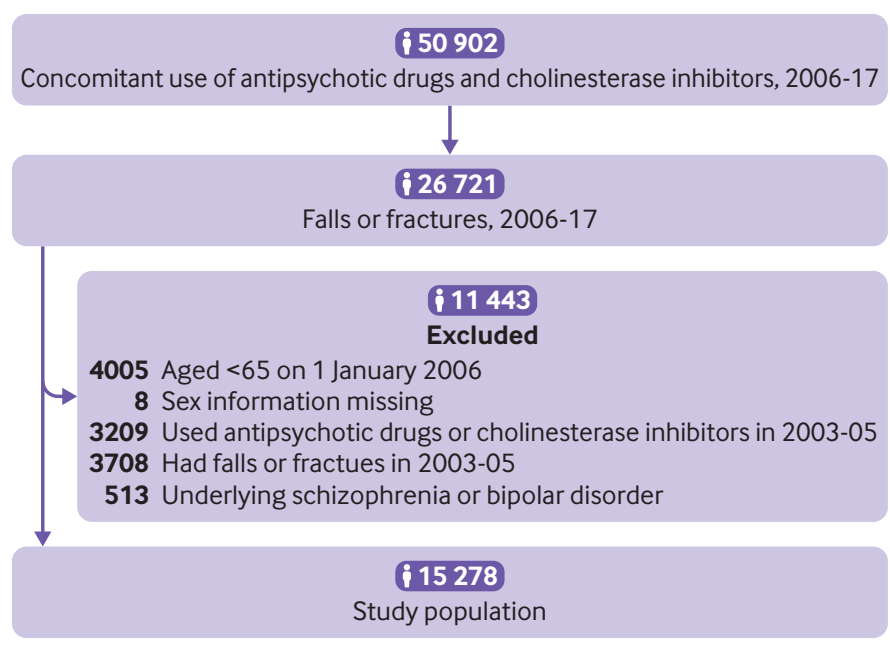

Fig 1 | Flowchart of study population selection

S92). We also analysed falls and fractures separately in secondary analyses. We only considered the first occurrence of an outcome in the analysis because recurrences of falls and fractures were not independent. Also, we included falls and fractures that required admission to hospital as a more severe outcome for a secondary analysis, which was defined by the primary diagnosis from the inpatient claims. We divided the study into five separate periods: 14 day pretreatment period before the use of drugs, use of cholinesterase inhibitors alone, use of antipsychotic drugs alone, use of a combination of cholinesterase inhibitors and antipsychotic drugs, and non-treatment period, when cholinesterase inhibitors and antipsychotic drugs were not used. The 14 day pretreatment period was designed to evaluate the increased incidence of falls and fractures related to the neuropsychiatric symptoms of major neurocognitive disorders before the start of drug treatment, and to benchmark the magnitude of the risk during the treatment periods. Figure 2 describes the five treatment periods. Continuous use of drugs was defined as patients refilling their prescriptions within 14 days after the end date of the last prescription (that is, 14 day grace period).

\section{Statistical analysis and covariates}

Means (standard deviations) or medians (interquartile ranges) are reported for continuous variables and numbers (percentages) for categorical variables. Baseline characteristics of the patients were assessed based on the covariates extracted from one year before 1 January 2006, including age, sex, subtype of major neurocognitive disorder, comorbidities, and co-administered drugs (table 1). Subtypes of major neurocognitive disorders included Alzheimer's disease, Parkinson's dementia, vascular dementia, mixed type (those with more than one diagnosis of a subtype of major neurocognitive disorders), and other, or unspecified..$^{38}{ }^{39}$ Supplementary table 1 lists the ICD codes for the subtypes of major neurocognitive disorders and comorbidities included in the study. Supplementary table 2 lists the ATC codes for the co- administered drugs included in the study. We also evaluated the characteristics of the patients at the time of the outcome events, including their age on the event date, where the event was diagnosed (outpatient, inpatient, or emergency department), mortality after the event, type of treatment, anticholinergic burden measured by the anticholinergic drug scale, and the ratio of prescribed daily dose to defined daily dose for cholinesterase inhibitors and antipsychotic drugs (table 2). The anticholinergic drug scale is a widely used measure developed through the expert opinion process, with a 4 point scale ranging from 0 , for no known activity, to 3three, for high anticholinergic activity. ${ }^{40}$ Prescribed daily dose is calculated as the sum of actual doses of each drug, whereas defined daily dose is a standard unit widely applied for the transition of drug doses with different strengths, developed by the World Health Organization. ${ }^{41}$ Defined daily dose is an assumed average maintenance dose per day for each drug.

We used the conditional Poisson regression model to calculate the incidence of falls and fractures in different study periods, and we generated the incidence rate ratios, with $95 \%$ confidence intervals, using the nontreatment period as the reference group. Self-controlled case series remove time constant confounders but are sensitive to time varying factors, such as the progression of major neurocognitive disorders. Therefore, we adopted a one year boundary to divide the study period to take into account possible effects related to age; that is, we divided the observation period by treatment status and also by one year intervals (eg, first, second, third years). This time varying method to adjust for the effects of age is described in detail elsewhere. ${ }^{37}$ Because some potentially important confounders, such as body weight, activities of daily living, and the use of walking aids, were not available in the database, we adopted a quantitative bias assessment tool, the $\mathrm{E}$ value approach, to evaluate the minimum effect from an unmeasured confounder that would be necessary to remove the association found between the treatment and the outcome. ${ }^{42}$ For example, an $\mathrm{E}$ value of 5 indicates that the unmeasured confounder would need to be associated with both treatment and outcome by a factor of more than five times to make the observed association irrelevant. Computation of the $\mathrm{E}$ value was based on Mathur et al. ${ }^{43}$

\section{Sensitivity analyses}

To examine the robustness of our findings, we conducted sensitivity analyses with different definitions of the study population, treatments, outcomes, and lengths of the pretreatment period. We grouped patients by sex (men or women) and age group (65-74, 75-84, and $\geq 85$ ) to examine any differential effects within these groups (supplementary tables 3-1 and 3-2). Anticholinergic burden (that is, the cumulative effect of taking multiple drugs with anticholinergic activities) is a critical issue that might also be associated with falls and fractures in elderly people. ${ }^{44} \mathrm{~A}$ high anticholinergic burden has been reported to adversely affect cognitive and 


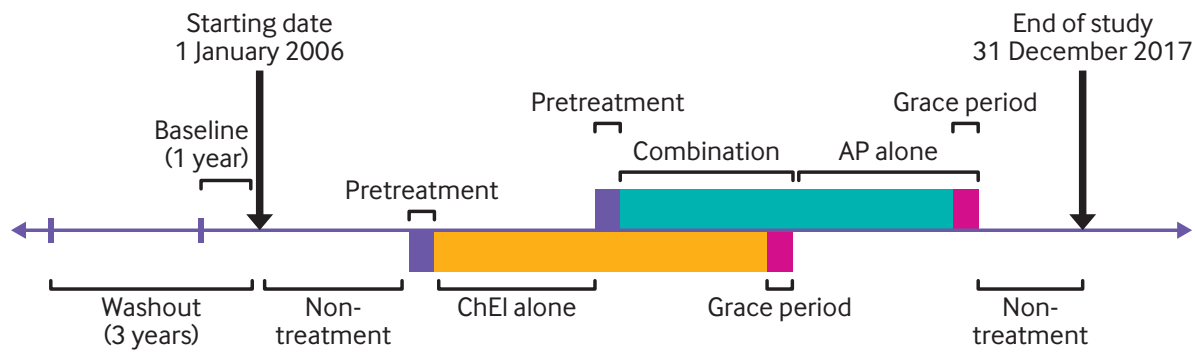

Fig 2 | Study scheme and definitions of treatment periods. A three year washout period before the start date was used to exclude patients with a history of falls and fractures. A one year baseline period was defined to assess patients' baseline covariates. The study was divided into five separate periods: 14 day pretreatment period before the use of drugs, use of cholinesterase inhibitors alone, use of antipsychotic drugs alone, use of a combination of cholinesterase inhibitors and antipsychotic drugs, and non-treatment period, when cholinesterase inhibitors and antipsychotic drugs were not used. A refill noted within 14 days after the end date of the last prescription (that is, 14 day grace period) was considered as a continuation of a previous prescription. $\mathrm{ChEl}=$ cholinesterase inhibitor; $\mathrm{AP}=$ antipsychotic drug

physical functions and might also increase the risk of falls, admission to hospital, and death. ${ }^{45-48}$ To account for the potential effect, we included the anticholinergic drug scale to classify patients by their anticholinergic burden for the sensitivity analyses (supplementary table 3-3). To examine the effect of dose, we grouped the study population based on their cumulative doses of antipsychotic drugs (above or below the median) with the ratio of prescribed daily dose to defined daily dose (supplementary table 3-4).

Patients in different care settings might have different baseline risks of falls and fractures. To understand the effect of the care setting, we conducted an analysis with outcomes restricted to those recorded only at outpatient settings to better reflect the risk in the community, where interventions to prevent falls and fractures might not be sufficient (supplementary table 3-5). To examine the effect of excluding schizophrenia and bipolar disorder, we conducted sensitivity analyses without exclusion of these conditions. We then further grouped this population by schizophrenia, bipolar disorder, and depression to examine the individual effects of these conditions (supplementary table 3-6).

Because patients who died from an outcome event would not have a subsequent treatment, potentially violating the assumption of self-controlled case series, we performed two sensitivity analyses after removing patients who died during the study period and patients who died within three months after the events (supplementary table 3-7). Moreover, to examine the validity of the outcomes we carried out a sensitivity analysis and selected specific diagnostic codes for falls and fractures (that is, falls from a different level (ICD-9 codes E880-E884; ICD-10 codes W00.1, W00.2, and W05-W17), falls from the same level (ICD-9 codes E885-E886; ICD-10 codes W00.0, W01-W04, and W18), and hip fractures (ICD-9 code 820; ICD-10 code S72)). Hip fractures were selected because more than $95 \%$ of such fractures were found to be related to falls $^{49}$ (supplementary table 3-8). In the main analysis, we included only the first incidence of falls or fractures because subsequent events might not be independent of previous falls or fractures. We performed a sensitivity analysis including all episodes of falls and fractures, however, to evaluate whether the exclusion of subsequent outcomes had substantially affected the results (supplementary table 3-9). We redefined the pretreatment period as seven, 21 , and 28 days to test the adequacy of a 14 day pretreatment period in the main analysis (supplementary table 3-10).

Each antipsychotic drug has different affinities to $\alpha$ adrenergic receptors, histamine receptors, and dopamine receptors, which can lead to varying degrees of effects that possibly provoke falls and fractures, such as a reduction in heart rate, vasodilation, orthostatic hypotension, blurred vision, sedation, and extrapyramidal symptoms. ${ }^{8}$ Therefore, we conducted subgroup analyses to evaluate the individual antipsychotic drugs separately. We selected haloperidol, risperidone, olanzapine, and quetiapine for the subgroup analyses because they were the most commonly used antipsychotic drugs for elderly patients in Taiwan (supplementary table 3-11). Some studies have found strong associations between antipsychotic drugs and falls and fractures immediately after the start of treatment or shortly after the end of treatment. ${ }^{17} 50$ We therefore defined two more study periods of 14 days: one after the start of treatment and the other after the end of treatment (supplementary table 3-12). We used SAS version 9.4 for all analyses.

\section{Patient and public involvement}

Patients and the public were not involved in this study because of the constrained situation during the covid-19 pandemic in Taiwan, as well as restrictions on funding.

\section{Results}

\section{Patient characteristics at baseline}

We identified 15278 adults eligible for the selfcontrolled case series (fig 1), with a mean age at baseline of 74.5 (standard deviation 5.5), of whom $66.7 \%$ were women (table 1). Although licensed neurologists or psychiatrists had reviewed all the diagnoses of major neurocognitive disorders, most of the study population had unspecified major neurocognitive disorders (10 570 patients, 69.2\%). The most common comorbidities were hypertension (7776, 50.9\%), 


\begin{tabular}{|c|c|}
\hline Variables & Study population $(n=15278)$ \\
\hline \multicolumn{2}{|l|}{ Sex: } \\
\hline Women & $10190(66.7)$ \\
\hline Men & $5088(33.3)$ \\
\hline Mean (SD) age (years) & $74.5(5.5)$ \\
\hline \multicolumn{2}{|l|}{ Age group: } \\
\hline $65-74$ & $7685(50.3)$ \\
\hline $75-84$ & $6966(45.6)$ \\
\hline$\geq 85$ & $627(4.1)$ \\
\hline \multicolumn{2}{|l|}{ Subtype of major neurocognitive disorders: } \\
\hline Alzheimer's disease & $3339(21.9)$ \\
\hline Parkinson's dementia & $990(6.5)$ \\
\hline Vascular dementia & $230(1.5)$ \\
\hline Mixed type (more than one subtype) & $149(1.0)$ \\
\hline Other or unspecified & $10570(69.2)$ \\
\hline \multicolumn{2}{|l|}{ Drug sequence: } \\
\hline Cholinesterase inhibitors before antipsychotic & $6688(43.8)$ \\
\hline Antipsychotic before cholinesterase inhibitors & $8289(54.2)$ \\
\hline Used concomitantly & $301(2.0)$ \\
\hline \multicolumn{2}{|l|}{ Comorbidities: } \\
\hline Depression & $932(6.1)$ \\
\hline Parkinson's disease & $287(1.9)$ \\
\hline Epilepsy & $60(0.4)$ \\
\hline Hypertension & $7776(50.9)$ \\
\hline Diabetes mellitus & $3347(21.9)$ \\
\hline Hyperlipidaemia & $3353(21.9)$ \\
\hline Coronary artery disease & $3229(21.1)$ \\
\hline Atrial fibrillation & $217(1.4)$ \\
\hline Heart failure & $589(3.9)$ \\
\hline Ischaemic stroke & $1573(10.3)$ \\
\hline Osteoarthritis & $4703(30.8)$ \\
\hline Osteoporosis & $1553(10.2)$ \\
\hline Cataract & $4261(27.9)$ \\
\hline \multicolumn{2}{|l|}{ Co-administered drugs: } \\
\hline Antidepressants & $1947(12.7)$ \\
\hline Psychostimulants & $861(5.6)$ \\
\hline Anxiolytics & $7904(51.7)$ \\
\hline Hypnotics and sedatives & $3337(21.8)$ \\
\hline Antiparkinsonian agents & $651(4.3)$ \\
\hline Anticonvulsants & $1178(7.7)$ \\
\hline Muscle relaxants & $6730(44.1)$ \\
\hline Vasodilators & $4993(32.7)$ \\
\hline Antihypertensive drugs & $1651(10.8)$ \\
\hline Diuretics & $3461(22.7)$ \\
\hline Renin-angiotensin-aldosterone system inhibitors & $4377(28.6)$ \\
\hline$\beta$ blockers & $4841(31.7)$ \\
\hline Calcium channel blockers & $5988(39.2)$ \\
\hline Antidiabetes drugs & $2801(18.3)$ \\
\hline Lipid modifying agents & $2631(17.2)$ \\
\hline Antiarrhythmic agents (classes 1 and 3 ) & $435(2.8)$ \\
\hline Antithrombotic agents & $5018(32.8)$ \\
\hline Steroids (systemic use) & $4238(27.7)$ \\
\hline Non-steroidal anti-inflammatory drugs & $11430(74.8)$ \\
\hline Bisphosphonates & $199(1.3)$ \\
\hline Parasympathomimetic drugs & $314(2.1)$ \\
\hline Antihistamines & $9734(63.7)$ \\
\hline
\end{tabular}

osteoarthritis (4703, 30.8\%), and cataract (4261, $27.9 \%)$ Non-steroidal anti-inflammatory drugs were the most commonly prescribed co-administered drugs (11430, 74.8\%), followed by antihistamines (9734, $63.7 \%)$, and anxiolytics $(7904,51.7 \%)$ (table 1).

Patient characteristics at time of event

Mean age on the event date was 79.7 (standard deviation 6.1) (table 2). About 7421 (48.6\%) patients had falls or fractures that required admission to hospital or a visit to the emergency department, and 264 (1.7\%) died within three months after the event. The median anticholinergic drug scale was 1.0 (interquartile range 3.0), and the median ratio of prescribed daily dose to defined daily dose, representing the cumulative antipsychotic dose, was 12.3 (49.0). Of the whole study population, 1960 (12.8\%) were taking cholinesterase inhibitors and 1374 (9.0\%) were taking antipsychotic drugs when the event occurred. The median dose of cholinesterase inhibitors ranged from 0.7 to 1.0 for the ratio of prescribed daily dose to defined daily dose, and the median dose of antipsychotic drugs ranged from 0.1 to 0.5 , similar to previous studies. ${ }^{51-53}$ Table 1 and table 2 shows the characteristics of the patients.

\section{Evaluation of the risk of falls and fractures}

Compared with the non-treatment period, the risk of falls and fractures was higher with the use of cholinesterase inhibitors alone (adjusted incidence rate ratio $1.17,95 \%$ confidence interval 1.10 to 1.24 ; Evalue 1.62), antipsychotic drugs alone (1.33, 1.24 to 1.43 ; E value 1.99), and the combination of cholinesterase inhibitors and antipsychotic drugs $(1.35,1.26$ to 1.45 ; E value: 2.04) (table 3). The risk was even higher during the pretreatment period $(6.17,5.69$ to 6.69 ; E value 11.82) compared with the non-treatment period. In the analysis of falls, the adjusted incidence rate ratios were 0.91 (95\% confidence interval 0.71 to 1.18 ), 1.36 (1.02 to 1.82 ), 1.55 (1.17 to 2.05), and 10.39 (8.08 to 13.37) for the use of cholinesterase inhibitors alone, antipsychotic drugs alone, the combination of cholinesterase inhibitors and antipsychotic drugs, and the pretreatment period, respectively. In the analysis of fractures, the adjusted incidence rate ratios were 1.18 (1.11 to 1.26 ), 1.34 (1.24 to 1.43 ), 1.35 (1.25 to 1.45$)$, and $6.11(5.62,6.63)$ for the use of cholinesterase inhibitors alone, antipsychotic drugs alone, the combination of cholinesterase inhibitors and antipsychotic drugs, and the pretreatment period, respectively. We included 7364 patients who had been admitted to hospital for falls or fractures, and the results were consistent with the main analysis (table 3)

\section{Sensitivity analyses}

The results from the sensitivity analyses were generally consistent with the main analysis. The use of cholinesterase inhibitors and antipsychotic drugs was associated with higher risks of falls and fractures compared with the non-treatment period, and the pretreatment period had a much higher risk of falls and fractures than any other period (table 4). Specifically, compared with the non-treatment period, we found that the adjusted incidence rate ratios for the pretreatment period were 2.81 (2.03 to 3.90), 6.11 (5.49 to 6.80), and 8.07 (7.03 to 9.26) for patients aged 65-74, 7584 , and $\geq 85$, respectively, and 7.63 (6.73 to 8.65) and 5.35 (4.80 to 5.95 ) for men and women, respectively. The adjusted incidence rate ratios of the pretreatment period were 5.30 (4.74 to 5.94) and 7.41 (6.60 to 8.33) in patients with an anticholinergic drug scale of $0-1$ 


\begin{tabular}{|c|c|}
\hline Variables & $\begin{array}{l}\text { Study population } \\
(n=15278)\end{array}$ \\
\hline Mean (SD) age on event date (years) & $79.7(6.1)$ \\
\hline \multicolumn{2}{|l|}{ Age group on event date: } \\
\hline $65-74$ & $3137(20.5)$ \\
\hline $75-84$ & $8703(57.0)$ \\
\hline$\geq 85$ & $3438(22.5)$ \\
\hline \multicolumn{2}{|l|}{ Outcome events: } \\
\hline Falls & $766(5.0)$ \\
\hline Falls from a different level & $200(1.3)$ \\
\hline Falls from the same level & $310(2.0)$ \\
\hline Fractures & $14874(97.4)$ \\
\hline Hip fractures & $2863(18.7)$ \\
\hline \multicolumn{2}{|l|}{ Setting of event being diagnosed: } \\
\hline Outpatient department & $7857(51.4)$ \\
\hline Inpatient department & $4644(30.4)$ \\
\hline Emergency department & $2777(18.2)$ \\
\hline Died during study period & $5198(34.0)$ \\
\hline Died within 3 months after event (No) & $264(1.7)$ \\
\hline Median (interquartile range) anticholinergic burden (anticholinergic drug scale) & $1.0(3.0)$ \\
\hline Median (interquartile range) cumulative dose of antipsychotic drugs (ratio of prescribed daily dose to DDD) & $12.3(49.0)$ \\
\hline \multicolumn{2}{|l|}{ Type of treatment on event date } \\
\hline Combination of cholinesterase inhibitors and antipsychotic drugs & $1270(8.3)$ \\
\hline Cholinesterase inhibitors alone: & $1960(12.8)$ \\
\hline Donepezil & $1826(12.0)$ \\
\hline Rivastigmine (oral) & $974(6.4)$ \\
\hline Rivastigmine (patch) & $242(1.6)$ \\
\hline Galantamine & $195(1.3)$ \\
\hline Antipsychotic drugs alone: & $1374(9.0)$ \\
\hline Haloperidol (oral) & $116(0.8)$ \\
\hline Haloperidol (parenteral) & $154(1.0)$ \\
\hline Risperidone & $401(2.6)$ \\
\hline Olanzapine & $110(0.7)$ \\
\hline Quetiapine & $1901(12.4)$ \\
\hline \multicolumn{2}{|l|}{ Median (interquartile range) dose on event date (ratio of prescribed daily dose to DDD) } \\
\hline \multicolumn{2}{|l|}{ Cholinesterase inhibitors: } \\
\hline Donepezil & $0.9(0.6)$ \\
\hline Rivastigmine (oral) & $0.7(0.4)$ \\
\hline Rivastigmine (patch) & $0.9(0.4)$ \\
\hline Galantamine & $1.0(0)$ \\
\hline \multicolumn{2}{|l|}{ Antipsychotic drugs: } \\
\hline Haloperidol (oral) & $0.1(0.2)$ \\
\hline Haloperidol (parenteral) & $0.1(0.2)$ \\
\hline Risperidone & $0.2(0.1)$ \\
\hline Olanzapine & $0.5(0.3)$ \\
\hline Quetiapine & $0.1(0)$ \\
\hline \multicolumn{2}{|l|}{ Median (interquartile range) time from the closest prescription to event (weeks) } \\
\hline \multicolumn{2}{|l|}{ Cholinesterase inhibitors: } \\
\hline Donepezil & $64.1(104.3)$ \\
\hline Rivastigmine (oral) & $59.7(123.0)$ \\
\hline Rivastigmine (patch) & $59.4(83.4)$ \\
\hline Galantamine & $80.9(146.5)$ \\
\hline \multicolumn{2}{|l|}{ Antipsychotic drugs: } \\
\hline Haloperidol (oral) & $7.9(44.0)$ \\
\hline Haloperidol (parenteral) & $0(2.3)$ \\
\hline Risperidone & $31.4(66.0)$ \\
\hline Olanzapine & $29.2(51.0)$ \\
\hline Quetiapine & $45.6(88.9)$ \\
\hline
\end{tabular}

and $\geq 2$ points, respectively. The adjusted incidence rate ratios of the pretreatment period were 5.73 (4.54 to 7.24 ) and 5.45 (4.54 to 6.53 ) in patients who had higher and lower cumulative doses of antipsychotic drugs, respectively.

Only 513 patients were excluded from the main analysis because of a diagnosis of schizophrenia or bipolar disorder, and when we re-selected the study population without excluding schizophrenia and bipolar disorder, the adjusted incidence rate ratio for the pretreatment period was 6.07 (5.60 to 6.57). The adjusted incidence rate ratios for the pretreatment period were 2.94 (0.70 to 12.37$)$ for patients with schizophrenia, 3.24 (1.76 to 5.99) for bipolar disorder, 


\begin{tabular}{|c|c|c|c|c|c|c|c|}
\hline & \multirow[b]{2}{*}{$\begin{array}{l}\text { No of } \\
\text { events }\end{array}$} & \multicolumn{2}{|c|}{ Follow-up time } & \multirow[b]{2}{*}{$\begin{array}{l}\text { Incidence rate }(95 \% \\
\mathrm{CI}) / 100 \text { person years }\end{array}$} & \multirow{2}{*}{$\begin{array}{l}\text { Crude incidence } \\
\text { rate ratio }(95 \% \\
\mathrm{Cl}) \text { compared with } \\
\text { non-treatment }\end{array}$} & \multicolumn{2}{|c|}{ Adjusted incidence rate ratio $(95 \% \mathrm{Cl})$} \\
\hline & & $\begin{array}{l}\text { Total person } \\
\text { years }\end{array}$ & $\begin{array}{l}\text { Median } \\
\text { (interquartile } \\
\text { range) years }\end{array}$ & & & $\begin{array}{l}\text { Compared with } \\
\text { non-treatment }\end{array}$ & $\begin{array}{l}\text { Compared with } \\
\text { pretreatment* }\end{array}$ \\
\hline \multicolumn{8}{|l|}{ All events $(n=15278)$ : } \\
\hline Non-treatment & 10208 & 122963.00 & $8.55(4.01)$ & $8.30(8.14$ to 8.46$)$ & Reference & Reference & $0.16(0.15$ to 0.18$)$ \\
\hline Pretreatment & 657 & 1254.90 & $0.07(0.03)$ & 52.35 (48.46 to 56.47$)$ & $6.31(5.83$ to 6.82$)$ & 6.17 (5.69 to 6.69$)$ & Reference \\
\hline Cholinesterase inhibitor alone & 1790 & 19018.33 & $0.96(1.92)$ & $9.41(8.98$ to 9.86$)$ & $1.13(1.08$ to 1.19$)$ & $1.17(1.10$ to 1.24$)$ & $0.19(0.17$ to 0.21$)$ \\
\hline Antipsychotic drug alone & 1353 & 13087.45 & $0.35(1.21)$ & 10.34 (9.80 to 10.89$)$ & $1.25(1.18$ to 1.32$)$ & $1.33(1.24$ to 1.43$)$ & $0.22(0.20$ to 0.24$)$ \\
\hline Combination & 1270 & 12037.35 & $0.56(1.31)$ & $10.55(9.98$ to 11.14$)$ & $1.27(1.20$ to 1.35$)$ & $1.35(1.26$ to 1.45$)$ & $0.22(0.20$ to 0.24$)$ \\
\hline \multicolumn{8}{|l|}{ Falls $(n=766)$ : } \\
\hline Non-treatment & 341 & 6750.84 & $9.28(3.32)$ & $5.05(4.54$ to 5.61$)$ & Reference & Reference & $0.10(0.07$ to 0.12$)$ \\
\hline Pretreatment & 84 & 62.84 & $0.07(0.03)$ & $134.70(107.30$ to 164.70$)$ & 26.46 (20.84 to 33.60) & 10.39 (8.08 to 13.37$)$ & Reference \\
\hline Cholinesterase inhibitor alone & 120 & 968.87 & $1.01(2.11)$ & $12.39(10.31$ to 14.76$)$ & 2.45 (1.99 to 3.02) & $0.91(0.71$ to 1.18$)$ & $0.09(0.06$ to 0.12$)$ \\
\hline Antipsychotic drug alone & 107 & 554.99 & $0.28(0.93)$ & 19.28 (15.88 to 23.20$)$ & $3.82(3.07$ to 4.74$)$ & $1.36(1.02$ to 1.82$)$ & 0.13 (0.09 to 0.18$)$ \\
\hline Combination & 114 & 518.55 & $0.49(1.16)$ & $21.98(18.22$ to 26.31$)$ & $4.35(3.52$ to 5.38$)$ & 1.55 (1.17 to 2.05$)$ & $0.15(0.11$ to 0.21$)$ \\
\hline \multicolumn{8}{|l|}{ Fractures $(n=14874)$} \\
\hline Non-treatment & 9998 & 119543.18 & $8.53(4.02)$ & $8.36(8.20$ to 8.53$)$ & Reference & Reference & $0.16(0.15$ to 0.18$)$ \\
\hline Pretreatment & 627 & 1222.01 & $0.07(0.03)$ & $51.31(47.41$ to 55.45$)$ & $6.13(5.66$ to 6.65$)$ & $6.11(5.62$ to 6.63$)$ & Reference \\
\hline Cholinesterase inhibitor alone & 1735 & 18491.49 & $0.96(1.91)$ & $9.16(8.74$ to 9.60$)$ & $1.12(1.07$ to 1.18$)$ & 1.18 (1.11 to 1.26$)$ & 0.19 (0.18 to 0.21$)$ \\
\hline Antipsychotic drug alone & 1299 & 12764.29 & $0.35(1.21)$ & $10.18(9.64$ to 10.74$)$ & $1.22(1.15$ to 1.29$)$ & $1.34(1.24$ to 1.43$)$ & $0.22(0.20$ to 0.24$)$ \\
\hline Combination & 1215 & 11759.94 & $0.56(1.31)$ & 10.33 (9.76 to 10.93$)$ & $1.24(1.16$ to 1.31$)$ & $1.35(1.25$ to 1.45$)$ & $0.22(0.20$ to 0.24$)$ \\
\hline \multicolumn{8}{|l|}{ Events in hospital $(n=7364)$ : } \\
\hline Non-treatment & 4382 & 58310.22 & $8.44(4.15)$ & $7.51(7.30$ to 7.74$)$ & Reference & Reference & $0.10(0.09$ to 0.11$)$ \\
\hline Pretreatment & 516 & 608.77 & $0.07(0.04)$ & $84.76(77.68$ to 92.32$)$ & $11.28(10.30$ to 12.36$)$ & $10.20(9.28$ to 11.21$)$ & Reference \\
\hline Cholinesterase inhibitor alone & 909 & 9005.44 & $0.94(1.85)$ & 10.09 (9.45 to 10.77$)$ & $1.34(1.25$ to 1.44$)$ & $1.27(1.17$ to 1.39$)$ & $0.12(0.11$ to 0.14$)$ \\
\hline Antipsychotic drug alone & 782 & 6584.79 & $0.36(1.26)$ & $11.88(11.07$ to 12.73$)$ & $1.58(1.46$ to 1.71$)$ & $1.55(1.41$ to 1.71$)$ & 0.15 (0.13 to 0.17$)$ \\
\hline Combination & 775 & 5905.85 & $0.57(1.34)$ & $13.12(12.22$ to 14.07$)$ & $1.75(1.62$ to 1.88$)$ & $1.73(1.57$ to 1.90$)$ & $0.17(0.15$ to 0.19$)$ \\
\hline
\end{tabular}

Combination=combination of cholinesterase inhibitors and antipsychotic drugs.

*Pretreatment period was considered as reference group and the analysis repeated.

and 4.13 (3.49 to 4.90) for depression. The confidence interval for the schizophrenia group was large because of the limited sample size $(\mathrm{n}=98)$. When we redefined the outcome by specific diagnosis codes, the adjusted incidence rate ratio for the pretreatment period was 10.16 (9.10 to 11.35$)$. When we redefined the length of the pretreatment period as seven, 21 , and 28 days, the adjusted incidence rate ratios for these pretreatment periods were 9.49 (8.64 to 10.43), 4.91 (4.56 to 5.30), and 4.43 (4.14 to 4.75 ), respectively. In the analyses focusing on individual antipsychotic drugs, we found that the adjusted incidence rate ratios were 3.75 (3.15 to 4.47$), 1.15$ (0.99 to 1.35$), 1.35$ (1.00 to 1.82 ), and 1.16 (1.07 to 1.26) with the use of haloperidol, risperidone, olanzapine, and quetiapine, respectively. Finally, the adjusted incidence rate ratios were 3.31 (2.96 to 3.70) within 14 days after the start of treatment and 1.24 (1.05 to 1.47) within 14 days after the end of treatment (supplementary table 3-12). Table 4 presents a summary of the results for the adjusted incidence rate ratios only. Supplementary tables 3-1 to 3-12 show the detailed results.

\section{Discussion}

In this population based self-controlled case series, we found that the use of cholinesterase inhibitors and antipsychotic drugs were both associated with a higher risk of falls and fractures compared with the non-treatment period, but the results should be looked at carefully. The 14 day pretreatment period showed a high incidence of falls and fractures, indicating that patients might have already been at high risk of outcome events before receiving treatment. The higher risks during the treatment periods, compared with the non-treatment period, could be because of the neuropsychiatric symptoms combined with the effects of the drugs. This finding implies that patients might still be in an unstable condition, despite receiving treatment. This conclusion remained robust throughout a series of subgroup and sensitivity analyses.

Previous studies have reported that cholinesterase inhibitors and antipsychotic drugs were associated with falls and fractures, with the magnitude of risk increasing from up to $18 \% \%^{5}$ to $63 \%{ }^{3}$ in patients receiving cholinesterase inhibitors, and from up to $21 \%{ }^{14}$ to $54 \%{ }^{22}$ in patients receiving antipsychotic drugs. Consistent with previous studies, our findings suggested that compared with thenon-treatment period, use of cholinesterase inhibitors and antipsychotic drugs increased the risk of falls and fractures by $17 \%$ and 33\%, respectively. This increased risk of falls and fractures with the use of cholinesterase inhibitors and antipsychotic drugs should be interpreted carefully, however. Our results showed that patients could have already been at high risk before receiving treatment, and the association between the patient's underlying conditions, the effects of the drugs in relieving the neuropsychiatric symptoms, and the side effects of the cholinesterase inhibitors and antipsychotic drugs makes it difficult to determine the exact contributing factors and to quantify the magnitude of the risk of falls and fractures that each factor poses. Similar findings have been reported in previous studies. Brännström et $\mathrm{al}^{26}$ reported that the highest risk of hip 


\begin{tabular}{|c|c|c|c|c|c|}
\hline & \multirow[b]{2}{*}{$\begin{array}{l}\text { No of } \\
\text { patients }\end{array}$} & \multicolumn{4}{|c|}{ Adjusted incidence rate ratio $\left(95 \%\right.$ confidence intervals) ${ }^{*}$} \\
\hline & & Pretreatment & $\begin{array}{l}\text { Antipsychotic drug } \\
\text { alone }\end{array}$ & $\begin{array}{l}\text { Cholinesterase } \\
\text { inhibitor alone }\end{array}$ & Combination \\
\hline Main analysis & 15278 & $6.17(5.69$ to 6.69$)$ & $1.33(1.24$ to 1.43$)$ & $1.17(1.10$ to 1.24$)$ & 1.35 (1.26 to 1.45$)$ \\
\hline \multicolumn{6}{|l|}{ Grouped by sex and age: } \\
\hline Men & 5088 & $7.63(6.73$ to 8.65$)$ & $1.55(1.38$ to 1.75$)$ & $1.18(1.06$ to 1.30$)$ & $1.48(1.31$ to 1.67$)$ \\
\hline Women & 10190 & 5.35 (4.80 to 5.95$)$ & $1.24(1.13$ to 1.35$)$ & $1.16(1.07$ to 1.25$)$ & $1.29(1.18$ to 1.41$)$ \\
\hline Age group $65-74$ & 3137 & 2.81 (2.03 to 3.90$)$ & $1.56(1.25$ to 1.93$)$ & $1.16(0.95$ to 1.40$)$ & $1.67(1.29$ to 2.16$)$ \\
\hline Age group 75-84 & 8703 & $6.11(5.49$ to 6.80$)$ & $1.28(1.17$ to 1.41$)$ & 1.18 (1.09 to 1.28$)$ & 1.25 (1.14 to 1.37$)$ \\
\hline Age group $\geq 85$ & 3438 & $8.07(7.03$ to 9.26$)$ & $1.20(1.05$ to 1.37$)$ & $1.29(1.15$ to 1.45$)$ & 1.51 (1.33 to 1.72$)$ \\
\hline \multicolumn{6}{|l|}{ Grouped by anticholinergic burden indicators: } \\
\hline Anticholinergic drug scale $\geq 2$ & 6579 & 7.41 (6.60 to 8.33$)$ & $1.76(1.60$ to 1.95$)$ & $1.35(1.24$ to 1.48$)$ & 1.67 (1.51 to 1.85$)$ \\
\hline Anticholinergic drug scale 0-1 & 8699 & $5.30(4.74$ to 5.94$)$ & $1.02(0.93$ to 1.13$)$ & 1.05 (0.96 to 1.13$)$ & 1.11 (1.01 to 1.23$)$ \\
\hline \multicolumn{6}{|l|}{ Grouped by cumulative dose of antipsychotic drugs: } \\
\hline Higher than median value & 3232 & $5.73(4.54$ to 7.24$)$ & $3.80(3.38$ to 4.27$)$ & $2.04(1.77$ to 2.35$)$ & 5.38 (4.79 to 6.05$)$ \\
\hline Equal to or lower than median value & 3247 & 5.45 (4.54 to 6.53$)$ & $2.91(2.54$ to 3.33$)$ & $1.66(1.48$ to 1.87$)$ & 2.12 (1.82 to 2.47$)$ \\
\hline Restricted to outcomes at outpatient settings only & 7914 & 2.54 (2.14 to 3.01$)$ & $1.12(1.01$ to 1.25$)$ & $1.09(1.00$ to 1.19$)$ & 1.02 (0.91 to 1.13$)$ \\
\hline $\begin{array}{l}\text { Re-selected patients without excluding schizophrenia and bipolar } \\
\text { disorder }\end{array}$ & 15791 & 6.07 (5.60 to 6.57$)$ & $1.32(1.24$ to 1.42$)$ & 1.17 (1.10 to 1.24$)$ & $1.33(1.24$ to 1.43$)$ \\
\hline Grouped by schizophrenia (yes) & 98 & $2.94(0.70$ to 12.37$)$ & 1.55 (0.80 to 2.99$)$ & 0.66 (0.16 to 2.72$)$ & 1.79 (0.75 to 4.28$)$ \\
\hline Grouped by schizophrenia (no) & 15693 & $6.08(5.61$ to 6.59$)$ & $1.32(1.23$ to 1.42$)$ & $1.17(1.10$ to 1.24$)$ & 1.33 (1.24 to 1.43$)$ \\
\hline Grouped by bipolar disorder (yes) & 426 & 3.24 (1.76 to 5.99$)$ & $1.12(0.80$ to 1.58$)$ & $1.19(0.79$ to 1.81$)$ & 0.84 (0.55 to 1.30$)$ \\
\hline Grouped by bipolar disorder (no) & 15365 & $6.15(5.67$ to 6.67$)$ & $1.33(1.24$ to 1.43$)$ & $1.17(1.10$ to 1.24$)$ & 1.35 (1.26 to 1.45$)$ \\
\hline Grouped by depression (yes) & 4743 & 4.13 (3.49 to 4.90$)$ & $1.12(1.00$ to 1.27$)$ & $1.03(0.91$ to 1.16$)$ & 1.06 (0.93 to 1.20$)$ \\
\hline Grouped by depression (no) & 11048 & 6.95 (6.34 to 7.61$)$ & $1.43(1.32$ to 1.56$)$ & $1.22(1.14$ to 1.31$)$ & $1.48(1.37$ to 1.61$)$ \\
\hline Removed patients who died during study period & 10080 & $6.43(5.80$ to 7.12$)$ & $1.25(1.15$ to 1.37$)$ & $1.10(1.02$ to 1.19$)$ & $1.18(1.08$ to 1.30$)$ \\
\hline Removed patients who died within three months after events & 15014 & $6.12(5.63$ to 6.64$)$ & $1.30(1.21$ to 1.40$)$ & $1.17(1.10$ to 1.24$)$ & $1.31(1.22$ to 1.41$)$ \\
\hline Redefined outcome by specific codes, all events & 5458 & $10.16(9.10$ to 11.35$)$ & $1.52(1.36$ to 1.69$)$ & $1.22(1.11$ to 1.35$)$ & $1.67(1.49$ to 1.87$)$ \\
\hline Falls & 621 & 10.77 (8.19 to 14.16$)$ & $1.26(0.91$ to 1.74$)$ & $0.87(0.65$ to 1.16$)$ & 1.49 (1.09 to 2.04$)$ \\
\hline Fractures & 5149 & $10.41(9.29$ to 11.66$)$ & $1.57(1.40$ to 1.76$)$ & $1.26(1.13$ to 1.39$)$ & $1.70(1.51$ to 1.91$)$ \\
\hline All episodes of falls and fractures & 15278 & $5.53(5.21$ to 5.88$)$ & $1.24(1.18$ to 1.31$)$ & $1.16(1.11$ to 1.21$)$ & $1.31(1.25$ to 1.38$)$ \\
\hline \multicolumn{6}{|l|}{ Redefined the length of pretreatment period to: } \\
\hline 7 days before treatment & 15278 & $9.49(8.64$ to 10.43$)$ & $1.30(1.21$ to 1.39$)$ & $1.17(1.10$ to 1.25$)$ & $1.32(1.23$ to 1.42$)$ \\
\hline 21 days before treatment & 15278 & $4.91(4.56$ to 5.30$)$ & $1.34(1.25$ to 1.44$)$ & $1.17(1.10$ to 1.24$)$ & $1.37(1.27$ to 1.47$)$ \\
\hline 28 days before treatment & 15278 & $4.43(4.14$ to 4.75$)$ & $1.35(1.26$ to 1.45$)$ & $1.18(1.11$ to 1.25$)$ & 1.39 (1.29 to 1.49$)$ \\
\hline \multicolumn{6}{|l|}{ Focus on individual antipsychotic drugs: } \\
\hline Haloperidol & 4745 & 12.11 (10.84 to 13.54$)$ & $3.75(3.15$ to 4.47$)$ & $1.16(1.06$ to 1.27$)$ & 4.26 (3.37 to 5.37$)$ \\
\hline Risperidone & 5119 & $2.74(2.23$ to 3.35$)$ & 1.15 (0.99 to 1.35$)$ & 1.23 (1.13 to 1.35$)$ & 1.32 (1.13 to 1.54$)$ \\
\hline Olanzapine & 1314 & $3.72(2.57$ to 5.37$)$ & 1.35 (1.00 to 1.82$)$ & 1.31 (1.09 to 1.56$)$ & 1.77 (1.31 to 2.39$)$ \\
\hline Quetiapine & 12851 & $5.40(4.91$ to 5.93$)$ & $1.16(1.07$ to 1.26$)$ & $1.18(1.11$ to 1.26$)$ & 1.16 (1.07 to 1.26$)$ \\
\hline
\end{tabular}

fractures occurred before the start of treatment with antipsychotic drugs (odds ratio 9.09, 95\% confidence interval 7.00 to 11.81 , for $16-30$ days before the start of treatment; and 5.84, 4.42 to 7.71 , for $1-15$ days before the start of treatment), rather than after receiving treatment $(4.31,3.05$ to 6.10 , within $1-15$ days). Pratt et $\mathrm{al}^{18}$ also found that the risk of admission to hospital for hip fractures was highest in the week before the start of treatment with antipsychotic drugs (incidence rate ratio 10.99 , 95\% confidence interval 7.94 to 15.21 ), and the risk was reduced within one week of starting treatment (1.04, 0.40 to 2.70$)$. These findings suggest that the higher risk of outcomes seen during the treatment periods might not be attributable to the drugs alone.

\section{Strengths and weaknesses of this study}

We used a large population based database to provide sufficient statistical power to evaluate the association between the drugs and potential adverse reactions. The nature of the self-controlled case series design allows controlling for time constant confounders by comparisons within individuals. ${ }^{37}$ We also adjusted the incidence rate ratios by a time varying method with regression models.

Our study had some limitations. Firstly, data on the severity of major neurocognitive disorders and valid diagnoses of neuropsychiatric symptoms were not available from the database, which might have caused confounding by indication. Also, our method of confirming the diagnoses of major neurocognitive disorders by the prescription records of cholinesterase inhibitors has not been validated. Secondly, we evaluated the risk of falls and fractures based on prescription records. We used a 14 day grace period to look at the residual effects of drugs after they were stopped, but misclassification bias is possible. Thirdly, we selected all diagnosis codes related to falls and fractures to ensure we had captured all possible outcome events from the database. Some of the codes, however, such as ICD-9 code E888 and ICD-10 code W19 (that is, unspecified falls), might not have been specific enough to reflect the relation between treatments and outcomes in this study. To evaluate 
the potential effect of these non-specific outcomes, we conducted a sensitivity analysis selecting only specific codes for falls and fractures. The results were consistent with those in the main analysis. Fourthly, some patients might have stopped taking the drugs because of minor falls or related symptoms, implying that those who continued with treatment represented a group of patients who tolerated the drugs well, possibly affecting the evaluation of the outcomes. Therefore, we performed a post hoc analysis to understand the extent of stopping treatment after a fall or a fracture. We found that only $7.9 \%$ of patients stopped their drug treatment after an event; thus stopping treatment and its subsequent effect on evaluation of the outcomes might be limited.

Another limitation of the study was that patients living in different care settings could have different baseline risks, which should be considered in the selfcontrolled case series. For example, the higher risk of outcomes during the pretreatment period might be because patients were living at home, with more trip hazards from rugs, stairs, and walking. Similarly, the lower risks during the treatment periods could have been partly because of the support from healthcare facilities. To assess the effect of the care setting, we conducted an additional analysis by including only outcome events recorded at outpatient settings. The results showed that the incidence rate ratios were smaller after limiting outcomes to only those that occurred in the community, but the risk for the pretreatment period remained higher than for the non-treatment period. Unmeasured confounders, such as patients' activities of daily living, could be another limitation of our study. The quantitative bias assessment (that is, E value) showed that for the potentially unmeasured confounders, a large effect size would be needed to refute the high risk of falls and fractures seen during the pretreatment period. According to the literature, ${ }^{1}$ potential unmeasured confounders have not been shown to have such a large effect size, and we thus concluded that the results were not affected significantly by these confounders. Not having the exact outcome dates for those who had falls or fractures when they were in hospital because the diagnoses were registered on the discharge date was also a limitation of our study, and thus the outcome dates we analysed could have been later than the dates when the event occurred. Therefore, we might have underestimated the risk during the treatment periods for patients who had falls or fractures in hospital and then stopped taking the drugs. Lastly, our study did not evaluate the dose-response relation between the drugs and the risk of falls and fractures. We compared the doses of antipsychotic drugs from our study population with those reported in guidelines and previous studies, however, and the doses were within the suggested ranges. ${ }^{51-53}$ Also, the group analysis by the ratio of prescribed daily dose to defined daily dose indicated that the doses of antipsychotic drugs did not have a differential effect on the risk of falls and fractures.
Implications of the study

Patients with major neurocognitive disorders often have cognitive impairment and neuropsychiatric symptoms that might cause substantial morbidity and mortality. ${ }^{2}$ Cholinesterase inhibitors can improve cognitive function, and antipsychotic drugs can control neuropsychiatric symptoms. Therefore, these drugs are commonly prescribed for patients with major neurocognitive disorders. Previous studies have suggested that the use of cholinesterase inhibitors ${ }^{34}$ and antipsychotic drugs ${ }^{10-22}$ could be associated with the risk of falls and fractures because of some of the side effects, such as hypotension, syncope, and extrapyramidal symptoms. Confounding by indication should be considered, however, because patients might already have been at high risk of falls and fractures before the treatment started. In our study, we found an increase in the risk of falls and fractures during the pretreatment period, which was reduced after patients received treatment. The risks during treatment were higher than during the non-treatment period, however. These findings suggest that close monitoring of early signs of falls and strategies for prevention are necessary during treatment.

From our sensitivity analyses, we identified subgroups that might have a higher risk of outcomes at baseline. Older patients and men had a higher risk of falls and fractures. Consistent with previous studies on the association of anticholinergic burden in elderly people and increased likelihood of falls and cognitive deterioration, ${ }^{54} 55$ we found a much higher risk during the pretreatment period in patients with a higher anticholinergic burden. A likely explanation for the higher risk with haloperidol could be that the associated extrapyramidal symptoms are greater than those of other antipsychotic drugs. Confounding by indication could be another explanation because patients with positive symptoms (eg, agitation) might be more likely to receive haloperidol. Also, we tested various pretreatment periods, from seven to 28 days. The incidence rate ratio was highest when the length of the pretreatment period was seven days (incidence rate ratio 9.49), and decreased as the length of the pretreatment period increased: incidence rate ratio was 6.17 at 14 days, 4.91 at 21 days, and 4.43 at 28 days. Based on these results, we conclude that a pretreatment period of seven days probably represents a period of rapid deterioration. On the other hand, because the status of patients with major neurocognitive disorders could be more severe when the pretreatment period is close to the start of treatment (eg, 0-7 days), defining a pretreatment period of more than 21 days possibly captures patients with a relatively more stable status. Therefore, our decision to use 14 days seems appropriate. The sensitivity analyses examined the robustness of the results and identified the effects of various definitions, and also provided variables for future studies. Moreover, the incidence rate ratio within 14 days after the start of treatment was higher than during other treatment periods, suggesting that for these people to become stable, a minimum duration 
of treatment might be required. The incidence rate ratio within 14 days after stopping treatment was higher than during the non-treatment period, suggesting that clinical attention is still necessary for the initial stage after patients stop their treatments.

\section{Unanswered questions and future research}

Although the reason for the increased risk of falls and fractures during the pretreatment period might be because patients are in an unstable condition, further studies are needed to confirm this theory. The risk during the treatment period could reflect a combination of patients' unsteadiness because of their disease, the effects of the drugs in relieving neuropsychiatric symptoms, and the side effects of the drugs. For example, some side effects of antipsychotic drugs, such as orthostatic hypotension, sedation, and extrapyramidal symptoms, could increase the risk of falls and fractures, whereas others, such as immobility, drowsiness, or being bedridden, could reduce the risk. These explanations are based mainly on clinical observations, however, and could not be exhaustively tested in our study. Future studies should consider the severity of major neurocognitive disorders (eg, minimental state examination or the clinical dementia rating scale) and patient reported information for a better understanding of how to deal with these issues.

\section{Conclusion}

The incidence of falls and fractures was especially high in the pretreatment period, suggesting that factors other than drugs, such as underlying diseases, should be taken into consideration when evaluating the association between the risk of falls and fractures and the use of cholinesterase inhibitors and antipsychotic drugs. The treatment periods were also associated with a higher risk of falls and fractures compared with the non-treatment period although the magnitude was much lower than during the pretreatment period. Strategies for prevention and close monitoring of the risk of falls are still necessary until there is evidence that patients have regained a more stable physical and mental state.

We thank Swu-Jane Lin for her critical comments on the manuscript, and the Health Data Science Center, National Cheng Kung University Hospital, for providing administrative support.

Contributors: GH-MW and EC-CL initiated the collaborative project, developed the research question, designed the study protocol, and drafted the manuscript. GH-MW and EC-CL are the guarantors. GH-MW, KKCM, W-HC, and EC-CL edited the study protocol, interpreted the results, and reviewed the manuscript. T-CL wrote the statistical analysis plan and conducted the statistical analysis. The corresponding author attests that all listed authors meet authorship criteria and that no others meeting the criteria have been omitted.

Funding: None.

Competing interests: All authors have completed the ICMJE uniform disclosure form at www.icmje.org/disclosure-of-interest/ and declare: EC-CL reports grants from the Ministry of Science and Technology of Taiwan (107-2320-B-006-070-MY3 and 106-2320-B-006-025MY2), outside the submitted work; KK-CM reports grants from the CW Maplethorpe Fellowship, National Institute of Health Research, UK, and the European Commission Horizon 2020 Framework, personal fees from IQVIA, and grants from Amgen and GlaxoSmithKline, outside the submitted work; no financial relationships with any organisations that might have an interest in the submitted work in the previous three years; no other relationships or activities that could appear to have influenced the submitted work.

Ethical approval: The study protocol was approved by the institutional review board of National Cheng Kung University Hospital (protocol No: B-ER-107-012).

Data sharing: We remotely accessed the data from the data centre of the Ministry of Health and Welfare. Individual level data are not available from the data centre. No additional data available.

The lead authors (the manuscript's guarantors) affirm that the manuscript is an honest, accurate, and transparent account of the study being reported; that no important aspects of the study have been omitted; and that any discrepancies from the study as planned (and, if relevant, registered) have been explained.

Dissemination to participants and related patient and public communities: We plan to write up a brief summary of the study with patients using plain language and then publish it on the official website of the School of Pharmacy, National Chen Kung University, Taiwan. We will also write a post on the official website of the National Cheng Kung University, which will tag all members in the research groups on Facebook and Twitter. We plan to submit our accepted manuscript to the National Cheng Kung University Hospital, National Cheng Kung University College of Medicine, and Taiwan Food and Drug Administration to apply for publication on their press releases. We will try to distribute the article to the official websites of other medical centres as well as to social media platforms, such as Facebook and Twitter.

Provenance and peer review: Not commissioned; externally peer reviewed.

This is an Open Access article distributed in accordance with the Creative Commons Attribution Non Commercial (CC BY-NC 4.0) license, which permits others to distribute, remix, adapt, build upon this work non-commercially, and license their derivative works on different terms, provided the original work is properly cited and the use is noncommercial. See: http://creativecommons.org/licenses/by-nc/4.0/.

1 Fernando E, Fraser M, Hendriksen J, Kim CH, Muir-Hunter SW Risk factors associated with falls in older adults with dementia: a systematic review. Physiother Can 2017;69:161-70. doi:10.3138/ ptc. 2016-14

2 Hefny AF, Abbas AK, Abu-Zidan FM. Geriatric fall-related injuries. Afr Health Sci 2016:16:554-9 doi:10.4314/ahs.v16i2.24

3 Epstein NU, Guo R, Farlow MR, Singh JP, Fisher M. Medication for Alzheimer's disease and associated fall hazard: a retrospective cohort study from the Alzheimer's Disease Neuroimaging Initiative. Drugs Aging 2014;31:125-9. doi:10.1007/s40266-013-0143-3

4 Ali TB, Schleret TR, Reilly BM, Chen WY, Abagyan R. Adverse effects of cholinesterase inhibitors in dementia, according to the pharmacovigilance databases of the United-States and Canada. PLoS One 2015;10:e0144337. doi:10.1371/journal.pone.0144337

5 Gill SS, Anderson GM, Fischer HD, et al. Syncope and its consequences in patients with dementia receiving cholinesterase inhibitors: a population-based cohort study. Arch Intern Med 2009;169:867-73. doi:10.1001/archinternmed.2009.43

6 Ohno Y, Kunisawa N, Shimizu S. Antipsychotic treatment of behavioral and psychological symptoms of dementia (BPSD): management of extrapyramidal side effects. Front Pharmacol 2019;10:1045. doi:10.3389/fphar.2019.01045

7 Tampi RR, Tampi DJ, Balachandran S, Srinivasan S. Antipsychotic use in dementia: a systematic review of benefits and risks from meta-analyses. Ther Adv Chronic Dis 2016;7:229-45. doi:10.1177/2040622316658463

8 UpToDate. Second-generation antipsychotic medications: Pharmacology, administration, and side effects; 2020. https:// www.uptodate.com/contents/second-generation-antipsychotic medications-pharmacology-administration-and-side-effects [cited 2020 Jul 27].

9 Samuel M, By the 2019 American Geriatrics Society Beers Criteria ${ }^{\circledR}$ Update Expert Panel. American Geriatrics Society 2019 Updated AGS Beers Criteria $®$ for potentially inappropriate medication use in older adults. J Am Geriatr Soc 2019;67:674-94. doi:10.1111/igs.15767

10 Leipzig RM, Cumming RG, Tinetti ME. Drugs and falls in older people: a systematic review and meta-analysis: I. Psychotropic drugs. J Am Geriatr Soc 1999;47:30-9. doi:10.1111/j.1532-5415.1999. tb01898x

11 Woolcott JC, Richardson KJ, Wiens MO, et al. Meta-analysis of the impact of 9 medication classes on falls in elderly persons. Arch Intern Med 2009:169:1952-60. doi:10.1001/archinternmed.2009.357

12 Bloch F, Thibaud M, Dugué B, Brèque C, Rigaud A-S, Kemoun G. Psychotropic drugs and falls in the elderly people: updated literature review and meta-analysis. J Aging Health 2011;23:329-46. doi:10.1177/0898264310381277 
13 Lapeyre-Mestre M. A review of adverse outcomes associated with psychoactive drug use in nursing home residents with dementia. Drugs Aging 2016;33:865-88. doi:10.1007/s40266-016-0414-x

14 Johnell K, Jonasdottir Bergman G, Fastbom J, Danielsson B, Borg N, Salmi P. Psychotropic drugs and the risk of fall injuries, hospitalisations and mortality among older adults. Int I Geriatr Psychiatry 2017;32:414-20. doi:10.1002/gps.4483

15 Seppala LJ, Wermelink AMAT, de Vries M, et al, EUGMS task and Finish group on fall-risk-increasing drugs. Fall-risk-increasing drugs: a systematic review and meta-analysis: II. Psychotropics. J Am Med Dir Assoc 2018;19:371.e11-7. doi:10.1016/j.jamda.2017.12.098

16 Liperoti R, Onder G, Lapane KL, et al. Conventional or atypical antipsychotics and the risk of femur fracture among elderly patients: results of a case-control study. J Clin Psychiatry 2007;68:929-34. doi:10.4088/JCP.v68n0616

17 Pouwels S, van Staa TP, Egberts AC, Leufkens HG, Cooper C, de Vries F. Antipsychotic use and the risk of hip/femur fracture: a populationbased case-control study. Osteoporos Int 2009;20:1499-506. doi:10.1007/s00198-008-0826-5

18 Pratt N, Roughead EE, Ramsay E, Salter A, Ryan P. Risk of hospitalization for hip fracture and pneumonia associated with antipsychotic prescribing in the elderly: a self-controlled caseseries analysis in an Australian health care claims database. Drug Saf 2011;34:567-75. doi:10.2165/11588470-000000000-00000

19 Lee S-H, Hsu W-T, Lai C-C, et al. Use of antipsychotics increases the risk of fracture: a systematic review and meta-analysis. Osteoporos Int 2017;28:1167-78. doi:10.1007/s00198-016-3881-3

20 Papola D, Ostuzzi G, Gastaldon C, et al. Antipsychotic use and risk of lifethreatening medical events: umbrella review of observational studies. Acta Psychiatr Scand 2019;140:227-43. doi:10.1111/acps.13066

21 Mortensen SJ, Mohamadi A, Wright CL, et al. Medications as a risk factor for fragility hip fractures: a systematic review and meta-analysis. Calcif Tissue Int 2020:107:1-9 doi:10.1007/s00223-020-00688-1

22 Koponen M, Taipale H, Lavikainen P, et al. Antipsychotic use and the risk of hip fracture among community-dwelling persons with Alzheimer's disease. J Clin Psychiatry 2017;78:e257-63. doi:10.4088/JCP.15m10458

23 Kim DH, Brown RT, Ding EL, Kiel DP, Berry SD. Dementia medications and risk of falls, syncope, and related adverse events: meta-analysis of randomized controlled trials. I Am Geriatr Soc 2011;59:1019-31. doi:10.1111/j.1532-5415.2011.03450.x

24 Jin B, Liu H. Comparative efficacy and safety of therapy for the behavioral and psychological symptoms of dementia: a systemic review and Bayesian network meta-analysis. J Neurol 2019;266:2363-75. doi:10.1007/s00415-019-09200-8

25 Epstein NU, Guo R, Farlow MR, Singh JP, Fisher M. Medication fo Alzheimer's disease and associated fall hazard: a retrospective cohort study from the Alzheimer's Disease Neuroimaging Initiative. Drugs Aging 2014:31:125-9 doi:10.1007/s40266-013-0143-3

26 Brännström J, Lövheim H, Gustafson Y, Nordström P. Antipsychotic drugs and hip fracture: associations before and after the initiation of treatment. J Am Med Dir Assoc 2020;21:1636-1642.e6. doi:10.1016/i.jamda.2020.06.047

27 McDonald WM. Overview of Neurocognitive Disorders. Focus (Am Psychiatr Publ) 2017;15:4-12. doi:10.1176/appi.focus.20160030.

28 Hsieh C-Y, Su C-C, Shao S-C, et al. Taiwan's national health insurance research database: past and future.Clin Epidemiol 2019;11:349-58. doi:10.2147/CLEP.S196293

29 Hsieh C-Y, Chen C-H, Li C-Y, Lai M-L. Validating the diagnosis of acute ischemic stroke in a National Health Insurance claims database. I Formos Med Assoc 2015;114:254-9. doi:10.1016/j.jfma.2013.09.009

30 Chen CC, Chen LS, Yen MF, Chen HH, Liou HH. Geographic variation in the age- and gender-specific prevalence and incidence of epilepsy: analysis of Taiwanese National Health Insurance-based data. Epilepsia 2012;53:283-90. doi:10.1111/j.1528-1167.2011.03332.x

31 Sung S-F, Hsieh C-Y, Lin H-J, Chen Y-W, Yang Y-HK, Li C-Y. Validation of algorithms to identify stroke risk factors in patients with acute ischemic stroke, transient ischemic attack, or intracerebral hemorrhage in an administrative claims database. Int Cardiol 2016;215:277-82. doi:10.1016/j.ijcard.2016.04.069

32 Lin YS, Chen TH, Chi CC, et al. Different implications of heart failure, ischemic stroke, and mortality between nonvalvular atrial fibrillation and atrial flutter-a view from a national cohort study. J Am Heart Assoc 2017;6:e006406. doi:10.1161/JAHA.117.006406

33 Liu C-C, Sun Y, Lee P-C, Li C-Y, Hu SC. Risk of dementia after Parkinson's disease in Taiwan: a population-based retrospective cohort study using National Health Insurance claims. BM Open 2019;9:e025274. doi:10.1136/bmjopen-2018-025274

34 Wu C-S, Kuo C-J, Su C-H, Wang SH, Dai H-J. Using text mining to extract depressive symptoms and to validate the diagnosis of major depressive disorder from electronic health records. J Affect Disord 2020;260:617-23. doi:10.1016/j.jad.2019.09.044
35 Wei MY, Luster JE, Chan C-L, Min L. Comprehensive review of ICD-9 code accuracies to measure multimorbidity in administrative data. $B M C$ Health Serv Res 2020;20:489. doi:10.1186/s12913-020-05207-4

36 Chien W-C, Lai C-H, Chung C-H, Lin C-H. A retrospective population-based data analyses of unintentional fall mortality and hospitalisation in Taiwan during 2005-2007. Int J Inj Contr Saf Promot 2013;20:50-8. doi:10.1080/17457300.2012.674042

37 Petersen I, Douglas I, Whitaker H. Self controlled case series methods: an alternative to standard epidemiological study designs. BMJ 2016;354:i4515. doi:10.1136/bmj.i4515

38 Lin P-C, Li C-H, Chou P-L, Chen Y-M, Lin L-C. Prevalence of pain-related diagnoses in patients with dementia: a nationwide study. J Pain Res 2018:11:1589-98, doi:10.2147/JPR.S172875

39 Wang H-K, Hung C-M, Lin S-H, et al. Increased risk of hip fractures in patients with dementia: a nationwide population-based study. BMC Neurol 2014:14:175. doi:10.1186/s12883-014-0175-2

40 Carnahan RM, Lund BC, Perry PJ, Pollock BG, Culp KR. The Anticholinergic Drug Scale as a measure of drugrelated anticholinergic burden: associations with serum anticholinergic activity. / Clin Pharmacol 2006;46:1481-6. doi:10.1177/0091270006292126

41 Leucht S, Samara M, Heres S, Davis JM. Dose equivalents for antipsychotic drugs: the DDD method. Schizophr Bull 2016:42(Suppl 1):S90-4. doi:10.1093/schbul/sbv167

42 VanderWeele TJ, Ding P. Sensitivity analysis in observational research: introducing the E-value. Ann Intern Med 2017;167:268-74 doi:10.7326/M16-2607

43 Mathur MB, Ding P, Riddell CA, VanderWeele TJ. Website and R package for computing E-values. Epidemiology 2018;29:e45-7. doi:10.1097/EDE.0000000000000864

44 Salahudeen MS, Duffull SB, Nishtala PS. Anticholinergic burden quantified by anticholinergic risk scales and adverse outcomes in older people: a systematic review. BMC Geriatr 2015:15:31. doi:10.1186/s12877-015-0029-9

45 Tune LE. Anticholinergic effects of medication in elderly patients. J Clin Psychiatry 2001;62(Suppl 21):11-4

46 Cao YJ, Mager DE, Simonsick EM, et al. Physical and cognitive performance and burden of anticholinergics, sedatives, and ACE inhibitors in older women. Clin Pharmacol Ther 2008;83:422-9. doi:10.1038/sj.clpt.6100303

47 Lowry E, Woodman RJ, Soiza RL, Mangoni AA. Associations between the anticholinergic risk scale score and physical function: potential implications for adverse outcomes in older hospitalized patients. J Am Med Dir Assoc 2011;12:565-72. doi:10.1016/j. jamda.2011.03.006

48 Uusvaara J, Pitkala KH, Kautiainen H, Tilvis RS, Strandberg TE. Association of anticholinergic drugs with hospitalization and mortality among older cardiovascular patients: A prospective study. Drugs Aging 2011;28:131-8. doi:10.2165/11585060000000000-00000

49 Parkkari J, Kannus P, Palvanen M, et al. Majority of hip fractures occur as a result of a fall and impact on the greater trochanter of the femur: a prospective controlled hip fracture study with 206 consecutive patients. Calcif Tissue Int 1999;65:183-7. doi:10.1007/s002239900679

50 Torstensson M, Leth-Møller K, Andersson C, Torp-Pedersen C, Gislason GH, Holm EA. Danish register-based study on the association between specific antipsychotic drugs and fractures in elderly individuals. Age Ageing 2017;46:258-64. doi:10.1093/ageing/afw209

51 Masopust J, Protopopová D, Vališ M, Pavelek Z, Klímová B. Treatment of behavioral and psychological symptoms of dementias with psychopharmaceuticals: a review. Neuropsychiatr Dis Treat 2018;14:1211-20. doi:10.2147/NDT.S163842

52 Haw C, Yorston G, Stubbs I. Guidelines on antipsychotics fo dementia: are we losing our minds?Psychiatr Bull 2009;33:57-60. doi:10.1192/pb.bp.107.017947

53 Lin Y-T, Hwang T-J, Shan J-C, Chiang H-L, Sheu Y-H, Hwu H-G. Dosage and duration of antipsychotic treatment in demented outpatients with agitation or psychosis. J Formos Med Assoc 2015;114:147-53. doi:10.1016/i.jfma.2012.09.002

54 Green AR, Reifler LM, Bayliss EA, Weffald LA, Boyd CM. Drugs contributing to anticholinergic burden and risk of fall or fall-related injury among older adults with mild cognitive impairment, dementia and multiple chronic conditions: a retrospective cohort study. Drugs Aging 2019;36:289-97. doi:10.1007/s40266-018-00630-z

55 Wilson NM, Hilmer SN, March LM, et al. Associations between drug burden index and falls in older people in residential aged care I Am Geriatr Soc 2011-59-875-80. doi:10.1111/j.1532 5415.2011.03386.x

Supplementary information: additional tables 1 to 3-12 and figure 1 